\title{
Hacerse hombre en el aula: masculinidad, homofobia y acoso escolar*
}

\section{Matías de Stéfano Barbero**}

\section{Resumen}

A partir del análisis de los datos producidos en el marco de la investigación Diversidad sexual y convivencia llevada a cabo entre 2013 y 2014 en diversos centros educativos españoles, el presente artículo reflexiona sobre el papel que juega la homofobia, como una forma específica de violencia, en la construcción, reproducción y control de la masculinidad entre los hombres jóvenes. Se sugiere que el acoso escolar es una forma ritualizada de violencia con una gran eficacia social entre los adolescentes, donde la homofobia, a través del miedo, invisibiliza y normaliza la violencia, silencia y aísla a las víctimas y perpetúa la legitimidad de las burlas, insultos y amenazas como una forma válida de relación entre pares.

Palabras clave: Masculinidad, Acoso Escolar, Homofobia, Violencia, LGBT.

* Recibido en 22 de febrero de 2016, aceptado en 8 de febrero de 2017.

** Becario CONICET - Instituto Interdisciplinario de Estudios de Género, Facultad de Filosofía y Letras, Universidad de Buenos Aires, Argentina. matiasdestefano@hotmail.com 
Manning Up in The Classroom: Masculinity, Homophobia and Bullying

\begin{abstract}
Based on the analysis of data produced in the framework of Diversidad sexual y convivencia research project, developed between 2013 and 2014 in different Spanish schools, this article reflects on the role of homophobia as a particular form of violence in the construction, reproduction and control of masculinity among young men. I suggest that bullying is a ritualized form of violence with a great social efficacy among adolescents, where homophobia, through fear, invisibilizes and normalizes violence, silence and isolate victims and perpetuates the legitimacy of teasing, insulting and threatening as valid forms of peer's relationship.
\end{abstract}

Keywords: Masculinity, Bullying, Homophobia, Violence, LGBT. 


\section{Introducción}

The first act of violence that patriarchy demands of males is not violence toward women. Instead patriarchy demands of all males that they engage in acts of psychic self-mutilation, that they kill off the emotional parts of themselves. If an individual is not successful in emotionally crippling himself, he can count on patriarchal men to enact rituals of power that will assault his self-esteem

(bell hooks, 2004:66).

Este artículo $^{1}$ tiene por objetivo reflexionar sobre la intersección entre homofobia y masculinidad en el acoso escolar en los centros educativos españoles, partiendo del análisis de los resultados del proyecto de investigación Diversidad sexual $y$ convivencia: Una oportunidad educativa ${ }^{2}$ (a partir de aquí DyC) en el que participé como investigador durante 2013 y 2014 y cuyos resultados fueron publicados en formato libro electrónico a finales del año 2015 (Pichardo, de Stéfano, 2015). Este proyecto comenzó ocho años después de la aprobación del matrimonio igualitario en España, con el objetivo principal de conocer en qué medida siguen presentes las situaciones de homofobia y transfobia en los centros educativos. En un primer análisis de los resultados, y en la

1 Quiero agradecer especialmente a José Ignacio Pichardo Galán por sus comentarios a este artículo.

2 El equipo de investigación fue coordinado por José Ignacio Pichardo Galán (Dpto. de Antropología Social de la Universidad Complutense de Madrid) e integrado por Matías de Stéfano Barbero (CONICET - Universidad de Buenos Aires), Mercedes Sánchez Sainz (Universidad Complutense de Madrid), Luis Puche Cabezas (Universidad Autónoma de Madrid), Belén Molinuevo Puras (Universidad de Syracuse Madrid y miembro del Área de Educación de la FELGTB) y Octavio Moreno Cabrera (Profesor de Educación Secundaria y miembro de la Asociación Transformarse para Transformar). El proyecto fue financiado por Google España y realizado con la colaboración de la Federación Estatal de Lesbianas, Gays, Transexuales y Bisexuales (FELGTB) y de la Asociación Transformarse para Transformar. 
línea de lo que ponen de manifiesto los últimos estudios realizados en España (Generelo, Pichardo y Galofré, 2008; Pichardo, 2009; Garchitorena, 2009; Generelo, 2012; Moreno y Puche, 2013), se advierte que el acoso escolar ${ }^{3}$ es una realidad incesante y que entre los principales motivos de burla, insulto y exclusión pueden distinguirse dos grupos. Por una parte, están ligados a las cuestiones de imagen tales como el sobrepeso y la no adecuación a los cánones de belleza; por otra, al cuestionamiento del sistema sexo-género, es decir, a ser o parecer lesbiana, gay, bisexual o transexual (LGBT), ser un "chico que se comporta como una chica" o "ser una chica que sale con muchos chicos"4. En estas páginas se ofrece un análisis de los datos de $D y C$ desde una perspectiva de género, poniendo especial atención en la configuración de los hombres como "sujetos genéricos" (Lagarde, 1996), para lo que utilizaremos el concepto instrumental "masculinidades" -cuyo origen situamos en las luchas de los feminismos y los movimientos LGBT-, no tanto para describir lo que los hombres jóvenes "son", sino para reflexionar sobre el género como elemento de la estructura social de desigualdades (Connell, 1995; Holter, 2005; Guasch, 2006; Kaufman, 2009). Esta perspectiva nos ofrece una lectura del acoso escolar como una forma de relación social mediada por la violencia que se articula de manera compleja entre los hombres jóvenes. La homosocialidad está estrechamente regulada por las normas, valores y conductas de la masculinidad hegemónica, donde la

3 Tanto en $D y C$ como en este artículo se utiliza la expresión "acoso escolar" para hacer referencia a las burlas, insultos, amenazas o situaciones de exclusión que se diferencian de las agresiones esporádicas por su intencionalidad, constancia y personalización, generando un desequilibrio de poder en las relaciones interpersonales (Del Barrio et al., 2003; De Stéfano, Pichardo, 2016).

4 Estas expresiones fueron utilizadas en el cuestionario para permitir al alumnado expresar el acoso sufrido con motivo de su expresión de género no normativa. Aún a riesgo de reproducir un binarismo que no refleja nuestros posicionamientos teóricos, en términos metodológicos consideramos que se trataba de la forma más clara y eficiente para no inducir a errores de comprensión a la hora de responder al cuestionario. 
homofobia interviene como una forma de control condicionando las relaciones y expresiones de género (Swain, 2005), llevando a los jóvenes a significar, percibir y reaccionar de formas particulares a la violencia de sus pares. Como señalan diversos autores (Leverenz, 1986; Blumenfeld, 1992; Parker, 1996; Kimmel, 1997; Borrillo, 2001; Pichardo, 2009) el acoso homófobo debe ser conceptualizado tanto en términos de género como de sexualidad, ya que cuando un joven heterosexual insulta a otro utilizando términos como "maricón" o "nenaza", lo hace no tanto aludiendo a la orientación sexual sino a una condición "no masculina" o "afeminada". Siguiendo este argumento, a lo largo de este artículo se irán abordando las especificidades del acoso escolar por homofobia (Generelo y Pichardo, 2006; Pichardo et al., 2015) e interrogando los datos producidos en el marco de $D y C$ para explorar en qué medida estas especificidades pueden ayudarnos a comprender y transformar la relación entre masculinidad y homofobia en el acoso escolar en España.

\section{Algunas precisiones teórico-metodológicas}

Este proyecto, llevado adelante por un equipo interdisciplinar, contó con una metodología mixta implementada en diversos puntos de España. En términos cuantitativos se recogieron 3236 cuestionarios respondidos por alumnado de ESO, bachillerato, $\mathrm{FP}$ y $\mathrm{PCPI}^{5}$ y 250 cuestionarios de profesorado de todos los niveles educativos no universitarios. ${ }^{6}$ La fase cualitativa

${ }^{5}$ En España, ESO es acrónimo de "Educación Secundaria Obligatoria" y comprende al alumnado entre 12 y 16 años y el bachillerato comprende al alumnado entre 16 y 18 años. FP es acrónimo de "Formación Profesional", programas destinados a la inserción, reinserción y actualización laboral para graduados de la ESO y del bachillerato. PCPI es acrónimo de "Programas de Cualificación Profesional Inicial" destinados a jóvenes entre 16 y 21 años que no hayan conseguido el título de ESO.

${ }^{6}$ Entre los centros con mayor participación destacan: IES Juan de la Cierva de Madrid (10 docentes y 866 estudiantes), Colegio Lourdes, primaria y secundaria, de Madrid (30 docentes y 397 estudiantes), IES Amurga de Gran Canaria (26 docentes y 343 estudiantes), IES Cabo Blanco de Tenerife (12 docentes y 310 
incluyó la realización y observación participante en 47 talleres sobre diversidad sexual y familiar impartidos en 6 centros de educación infantil y primaria ${ }^{7}$, así como 11 entrevistas en profundidad al profesorado, alumnado y familiares recogiendo sus experiencias relativas a la diversidad sexual y familiar en las aulas. Todos los datos producidos en esta primera fase de la investigación fueron recogidos y tratados de forma anónima y confidencial. Finalmente, en una segunda y última fase de este proyecto, el equipo ha realizado 12 entrevistas en profundidad a jóvenes lesbianas, gays, bisexuales, transexuales (LGBT) y asexuales, donde relatan sus experiencias y estrategias al haber decidido ser visibles y apostar por el respeto a la diversidad sexual en diferentes ámbitos de su vida. ${ }^{8}$

En este punto es necesario precisar que los datos producidos para este proyecto no son estadísticamente representativos del conjunto del alumnado en España. Debido a las limitaciones presupuestarias, sólo se ha podido acceder al profesorado a través de la metodología conocida como "bola de nieve", implementada entre las redes personales y profesionales de los miembros del equipo de investigación y de la difusión del proyecto realizada por la FELGTB, la Federación de Enseñanza de Comisiones Obreras (FECCOO) y la Asociación Transformarse para Transformar. Aun así, los resultados de $D y C$ son similares a estudios semejantes

estudiantes), IES Carmen Martín Gaite de Madrid (6 docentes y 267 estudiantes), IES Las Breñas de La Palma (4 docentes y 146 estudiantes), IES Virgen de la Paz de Madrid (15 docentes y 112 estudiantes), CEIP Valdés Leal de Sevilla (11 docentes).

${ }^{7}$ Los talleres fueron realizados en los siguientes centros educativos: CEIP Valdés Leal (Sevilla), CEIP Andalucía (Sevilla), CEIP Duque de Alba (Loeches, Madrid), Colegio Santa Cristina FUHEM (Madrid), Colegio Ntra. Señora de Lourdes, FUHEM (Madrid), CEIP Federico García Lorca (Colmenar Viejo, Madrid).

8 Las entrevistas fueron grabadas en video y posteriormente se realizó con ellas un audiovisual [disponible en http://presentacionidyc.blogspot.com/]. 
realizados a nivel nacional $e$ internacional ${ }^{9}$, y han demostrado ser más que significativos. ${ }^{10}$

Cómo ya se ha mencionado, la siguiente interpretación de los datos producidos durante el proyecto parte del marco teórico de los estudios de género, especialmente sobre masculinidades. Para ello, se han desagregado los datos distinguiendo entre sexos, poniendo especial atención a que ello, por sí solo, no sólo no nos asegura la aplicación de una perspectiva de género, sino que incrementa el riesgo de esencializar y homogeneizar las masculinidades (Núñez, 2009; de Stéfano, 2015). Es por ello que también se han desagregado e interpretado los datos teniendo en cuenta la diversidad sexual y las relaciones de género respectivamente.

Finalmente, es importante mencionar que los datos utilizados en este artículo para reflexionar sobre la intersección entre masculinidad y homofobia en el acoso escolar parten de un proyecto de investigación que no ha sido diseñado especialmente con ese objetivo, por lo tanto, más que ofrecer respuestas explicativas, se ofrecen reflexiones que pueden ayudarnos a comprender la complejidad del fenómeno del acoso escolar por homofobia en su intersección con el género y las masculinidades.

\section{El acoso escolar como un "juego de hombres"}

De acuerdo a los resultados de $D y C$, la mayor parte del alumnado y del profesorado coinciden en que el ambiente de convivencia de sus centros educativos es tranquilo. Sin embargo,

9 Recogidos y analizados por Belén Molinuevo en el capítulo "Una mirada internacional a las buenas prácticas del profesorado frente a la homofobia", pp. 12-19 de Diversidad sexual y convivencia: Una oportunidad educativa (2015).

${ }^{10}$ Hemos reflexionado sobre el proceso metodológico y la difusión de los resultados de este proyecto de investigación en De Stéfano, M.; Puche, L.; Pichardo, J.I. (2015) "El compromiso de la investigación social en la construcción de otra escuela posible" en Revista Interuniversitaria de Formación del Profesorado $\mathrm{n}^{\circ} 82$ (29.1), Abril 2015. Universidad de Zaragoza: España. ISSN: 0213-8646. Enlace: http://www.redalyc.org/articulo.oa?id=27439665004 
sólo 1 de cada 10 estudiantes afirma no haber presenciado burlas, insultos, exclusión, amenazas o agresiones en su centro educativo. Algo más de la mitad señala haberlas presenciado alguna vez $(56 \%)$ y casi una tercera parte, a menudo y constantemente (18,39\% y $10,60 \%$ respectivamente). Para comprender la serie de relaciones que subyace a esta aparente contradicción, y como veremos a lo largo del artículo, el género es una variable central, ya que hombres y mujeres jóvenes perciben la violencia y se relacionan con ella de manera diferente.

Si bien casi la mitad del alumnado participante en la investigación (46\%) afirma no haber insultado, burlado o excluido a sus pares, casi el mismo número $(45,5 \%)$ afirma haberlo hecho alguna vez $(42,2 \%)$, a menudo $(2,1 \%)$ o constantemente $(1,2 \%)$. Es aquí donde una primera lectura con perspectiva de género nos ofrece datos reveladores. Como se señala en el gráfico siguiente, los hombres jóvenes respondieron de forma muy diferente a las mujeres en lo relativo a la frecuencia con la que insultan, burlan o excluyen a sus pares. Los chicos duplican el porcentaje de chicas que afirman acosar constantemente, lo triplican en la frecuencia "a menudo" y superan en $10 \%$ a aquellas que lo hacen alguna vez. Por su parte, aquellas que nunca han acosado al resto de alumnado superan en un $14 \%$ a los chicos.

\section{Frecuencia por sexos con la que se insulta, burla o excluye}

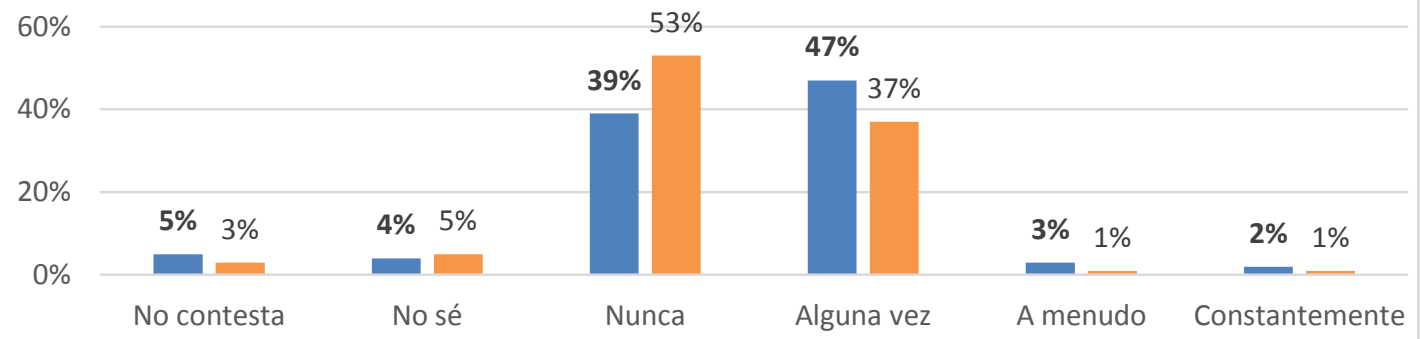


El hecho de que los hombres son los que predominan a lo largo de todo el espectro de la violencia no es una novedad (Connell, 2000), y el acoso escolar no es una excepción. Pero, ¿̇por qué los hombres son más violentos que las mujeres? A lo largo de la historia, han existido multitud de explicaciones. La sociobiología, particularmente, ha alimentado el sentido común extendiendo la idea de que la violencia es "natural" en los hombres (Connell, 1995; Kaufman, 2009). Sin embargo, no existen evidencias claras de que así sea, sino que todo apunta a que se ha ido naturalizando a través del sesgo androcéntrico que caracteriza al pensamiento científico, y que ha sido puesto al servicio de los intereses patriarcales para justificar y legitimar la violencia masculina (Guasch, 2006). Hoy sabemos que los niveles de testosterona, por ejemplo, no tienen resultados significativos sobre el comportamiento si no es en combinación con estímulos sociales específicos vinculados al género (Kemper, 1990). Por otra parte, recientes investigaciones (Joel et al., 2015) revelan que los cerebros humanos, aun cuando muestran diferencias vinculadas al sexo, no pueden distinguirse claramente entre "masculinos" y "femeninos", sino que cada uno se encuentra en un contínuum entre esos extremos y presenta diferencias individuales en su composición específica. Por su parte, los estudios transculturales sobre la diversidad de configuraciones de las masculinidades (Gilmore, 1991), han revelado que a lo largo y lo ancho del mundo existen sociedades en las que los hombres no son violentos con las mujeres o los niños y niñas, ni siquiera los unos con los otros, demostrando que la biología no es la matriz explicativa única de la violencia.

Para comprender los actos de violencia debemos analizarlos dentro de los contextos sociales en los que tienen lugar (Kaufman, 2009). En nuestras sociedades, la violencia es una constante presente en diversos aspectos de la vida social, económica, política, ambiental, afectiva y corporal, adquiere formas físicas, psicológicas y simbólicas, y se institucionaliza cristalizando en formas de opresión estructuradas: las raíces del sexismo, la heteronormatividad, el clasismo y el racismo -entre otras- se 
intersectan en cada acto de violencia específico (Viveros, 2011). Las distintas formas de violencia son una epidemia de las relaciones humanas, pero para responder al porqué de su prevalencia entre los hombres debemos interrogar la serie de relaciones de poder que se ponen en juego.

Social y culturalmente, exaltamos un tipo de masculinidad que influencia poderosamente la vida de los hombres. Desde pequeños, a través de los proceso de socialización, les enseñamos a distinguir entre la actividad y la pasividad, la autosuficiencia y la dependencia, la razón y la emoción, la fortaleza y la debilidad, el honor y la vergüenza, la valentía y la cobardía, el éxito y el fracaso, la dominación y la subordinación. Mientras que los primeros términos de estas dicotomías se construyen como deseables y masculinos, los segundos definen la otredad femenina, $y$ es mostrando una férrea oposición a ella que los hombres construyen su identidad. Pero la violencia masculina no está únicamente dirigida a la dominación y control de las mujeres, sino también de otros hombres e incluso de uno mismo, como señala el epígrafe de bell hooks (2004) que da inicio a este artículo.

Es por ello que en el acoso escolar encontramos que los hombres jóvenes dirigen las burlas $e$ insultos contra sus compañeras mujeres (40\%), pero incluso en mayor medida contra sus propios compañeros varones $(62,59 \%)$. Además, las mujeres jóvenes sufren menos acoso que los hombres y también lo perpetran en una significativa menor medida. 
Relación entre quien sufre y quién efectúa el acoso según su sexo

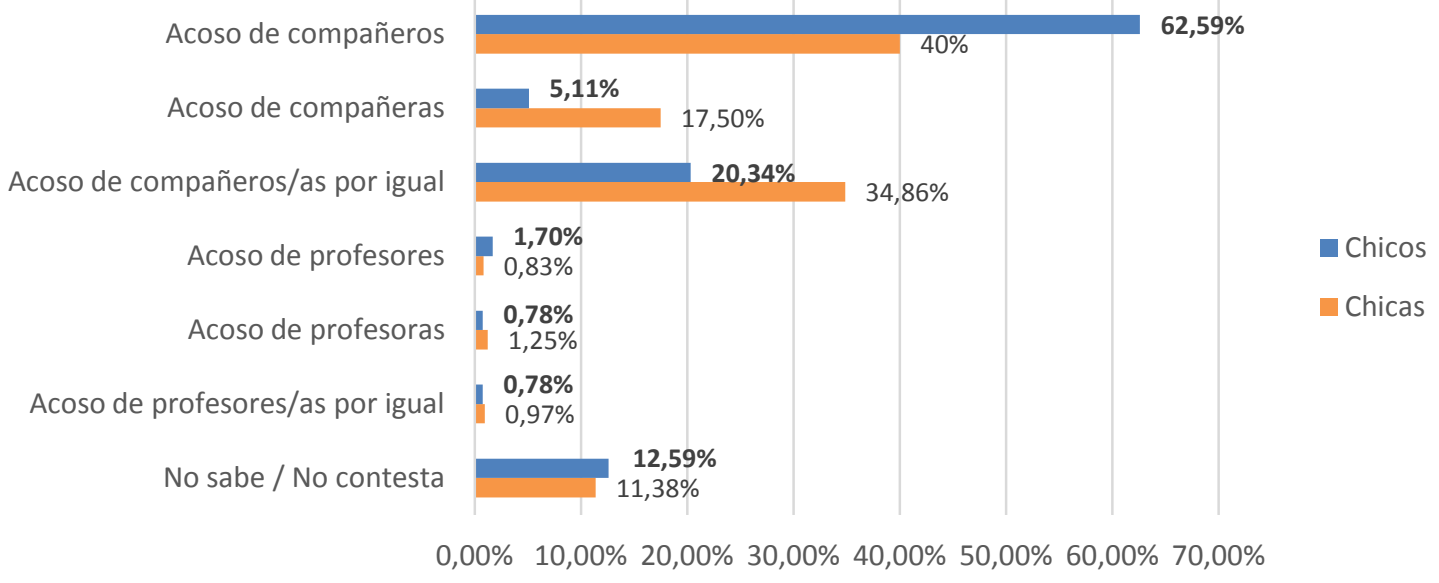

De acuerdo con Connell (1995, 2005), existe una multiplicidad de masculinidades atravesadas por relaciones de poder donde se pueden identificar dos formas de hegemonía: la externa, de dominación masculina sobre las mujeres -nótese en los datos del cuadro anterior que el acoso escolar que sufren las chicas también es perpetrado mayoritariamente por varones- y la interna, de dominación de unos hombres sobre otros. Es en la pugna por la legitimidad y el poder masculino donde se configura la masculinidad hegemónica, entendida como "la configuración de la práctica de género que incorpora la respuesta aceptada, en un momento específico, al problema de la legitimidad del patriarcado, lo que garantiza (o se considera que garantiza) la posición dominante de los hombres y la subordinación de las mujeres" (Connell, 1995:117). Así, existe una pluralidad de configuraciones de las masculinidades cuyas relaciones de poder están atravesadas por la violencia, pero también, y como iremos viendo, por formas de persuasión, complicidad y miedo.

Hasta aquí, el acoso escolar se presenta como un "juego de hombres" (Seidler, 2009), pero no sólo porque ellos sean quienes 
más perpetran y sufren la violencia, sino porque es en los puntos críticos de confrontación y respuesta donde se pone en juego la masculinidad, y de ello depende que salga fortalecida o cuestionada. Esto se debe al miedo de los hombres jóvenes a ser "avergonzados o humillados delante de otros hombres, [a] ser dominados por hombres más fuertes" (Leverenz, 1986:451), a ver cuestionada su masculinidad al dejar sin respuesta la confrontación. La masculinidad es poder pero, paradójicamente, es frágil y quebradiza. Al igual que la violencia, no radica en la biología, sino que es una ideología que debe ser constantemente performada y demostrada, con tal intensidad, que parezca inevitable, natural, biológica (Connell, 1995, 2000; Kimmel, 1997; Kaufman, 2009). Por ello, los hombres esconden una inseguridad pasmosa que cargan con una tensión casi constante: al mismo tiempo, la masculinidad es atractiva, al ser una promesa de poder, de éxito; pero resulta igualmente amenazante, ya que implica la automutilación emocional y la constante vigilancia de uno mismo (Tolson cit. Kaufman, 2009:587). Esto es especialmente evidente durante la adolescencia, cuando la represión y ocultamiento de todas las características consideradas femeninas es especialmente vigilada por otros hombres. Esta tensión no puede ser revelada, ni aliviada por el llanto o la conversación, ya que se pondría en peligro la masculinidad: a medida que avanza el desarrollo de los niños, la violencia se presenta como el único medio disponible para sublimar la tensión (Kaufman, ibíd.). Un joven de 17 años que declara burlar e insultar constantemente lo expresa así: "La mayoría de los insultos son coñas, yo llevo desde $1^{\circ}$ de la ESO soportando insultos como 'gorda', 'vaca', 'foca', etc... Pero al igual yo contesto y llega un punto en el que nos lo tomamos a coña todos". Esta es sólo una de las referencias que el alumnado, en el espacio reservado en el cuestionario para las respuestas espontáneas, hace sobre la violencia verbal (18 casos) y la violencia física (28 casos) como formas de respuesta a las situaciones de acoso.

Como se señala en el siguiente gráfico, el porcentaje de hombres jóvenes que al mismo tiempo sufren y perpetran acoso 
escolar es significativamente mayor al porcentaje de mujeres jóvenes. Los hombres jóvenes que han sufrido acoso escolar y afirman ser ellos mismos acosadores "constantemente" y "a menudo" triplican al número de mujeres jóvenes y las supera en un $20 \%$ en la frecuencia "alguna vez". Por su parte, el porcentaje de ellas que aun sufriendo acoso escolar nunca ha acosado, es casi un $20 \%$ superior a los hombres jóvenes que nunca han respondido con violencia.

\section{Porcentaje de jóvenes por sexo que han sufrido acoso \\ y que también han acosado}

Víctimas que han acosado "Constantemente"

Víctimas que han acosado "A menudo"

Víctimas que han acosado "Alguna vez"

Víctimas que no han acosado "Nunca"

Víctimas que no saben / no contestan

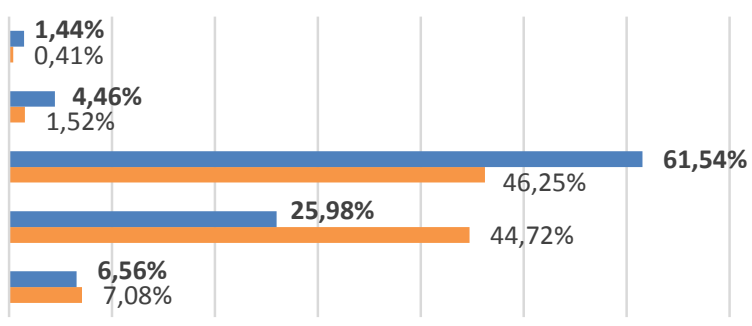

0,00\% 10,00\% 20,00\% 30,00\% 40,00\% 50,00\% 60,00\% 70,00\%

Chicos Chicas

Si la masculinidad es un terreno de disputa, este "juego de hombres" necesita de un escenario donde esté presente el público que aprecie la performance masculina. La masculinidad debe ser aprobada y validada homosocialmente: los hombres se vigilan y juzgan concediéndose la aceptación o el rechazo en el reino de la hombría (Kimmel, 1997). Como se señala en el siguiente gráfico, existen tres lugares de riesgo especialmente señalados en los centros educativos en lo relativo al acoso escolar: el momento del cambio de clases, el patio de recreo y durante las clases. Así, vemos que el acoso escolar no tiene lugar en espacios privados o reservados, sino que sucede sobre todo en espacios donde el resto del alumnado -pero también el profesorado- está presente. 


\section{Relación por sexos de lugares donde se sufre acoso escolar}

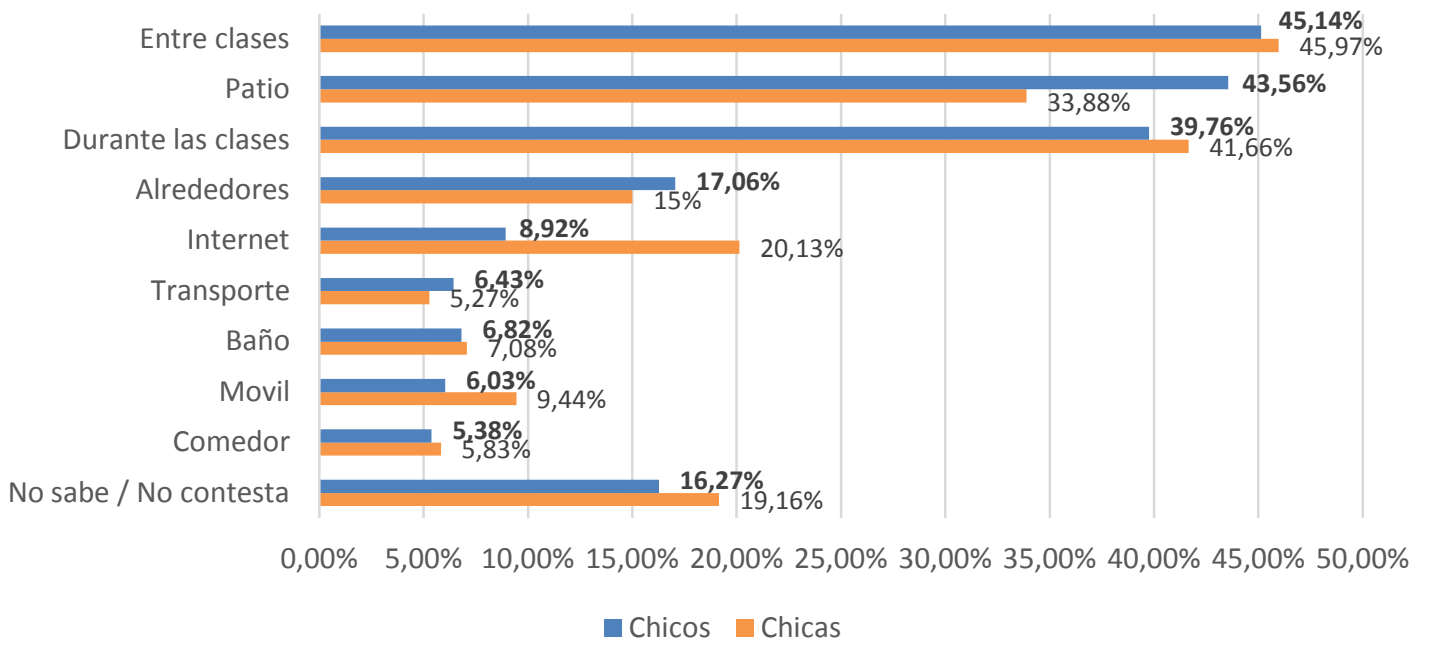

No sería extraño que los hombres que estén leyendo estas páginas recuerden sus experiencias escolares y consigan identificarse con estas situaciones. Todos aprendimos en la infancia y la adolescencia a burlar, insultar y pelear, a hacer chistes o a huir para evitar una pelea... De alguna forma u otra, todos nos vimos forzados a aprender a gestionar la violencia. Yo mismo recuerdo los partidos de "fútbol rudo" que jugábamos en los recreos con una lata de gaseosa aplastada. En un colegio sólo de varones, esta parecía ser una excelente estrategia para dirimir las tensiones del grupo en un contexto preparado para legitimar la violencia y validar frente a otros hombres la propia masculinidad. Cuando las tensiones no encontraban una vía de escape alternativa, el ultimátum llegaba en forma de frase lapidaria: "te espero a la salida". Con ella, el ritual de violencia escolar adolescente llegaba a su cumbre y comenzaban los preparativos 
para la ceremonia donde una multitud de jóvenes reunidos en círculo rodearía entre arengas a los contendientes que se disputarían a golpes el trofeo de una masculinidad reforzada por el prestigio otorgado por el grupo de pares.

Una vez presentadas estas primeras características de este "juego de hombres", en el próximo apartado veremos cómo las especificidades de la homofobia se intersectan con la construcción de la masculinidad en el acoso escolar.

\section{Invisibilización, normalización, miedo y silencio}

Como hemos señalado hasta aquí, sexo y género son variables que pueden ayudarnos a comprender algunas especificidades del acoso escolar. Sin embargo, veremos a continuación cómo su intersección con la sexualidad nos lleva a advertir las posibilidades que una perspectiva desde las masculinidades nos ofrece para emprender estrategias en la prevención y erradicación del acoso escolar.

La infancia y la adolescencia son etapas del desarrollo humano en las que se juega, experimenta y negocia constantemente con el género y la sexualidad (Connell, 2002:1216). Sin embargo, el juego, la experimentación y la negociación que involucran a estas cuestiones tienen reglas y consecuencias diferentes a lo largo de las etapas educativas. De acuerdo con la observación participante realizada en los talleres ofrecidos en la etapa de educación infantil, las primeras burlas e insultos no se encuentran vinculados con el género ni con la sexualidad, sino que giran en torno a las cuestiones físicas relativas al peso y la altura. Las observaciones del equipo de investigación y las experiencias del profesorado coinciden en que es precisamente en los primeros años de la etapa de educación primaria en la que aparecen insultos como "mariquita" o "marimacho" dirigidos a aquellos niños que "hacen cosas de niñas" y viceversa. Como señala Kaufman (2009:586), es a los 5 o 6 años de edad cuando comienzan a establecerse las bases de la masculinidad, y no es sino hasta el último ciclo de primaria que se aprecia que estos 
insultos se relacionan con la orientación sexual. De acuerdo con el autor, antes de los 6 años la masculinidad lleva tiempo formándose pero aún permanece latente, comienza a fortalecerse y expresarse en el desarrollo del niño y termina por explotar en la adolescencia, donde el rechazo a "la feminidad" y la pasividad se hacen ya evidentes, $y$ hacen que la mayor parte de los hombres jóvenes responda a la violencia con violencia, reforzando los baluartes de la masculinidad (ibíd.:586-587).

En resumen, a medida que se avanza a través del sistema educativo, las cuestiones relativas a los roles, expresiones e identidades de género primero, y más tarde a la orientación sexual, comienzan a transformarse, pasando del juego y la experimentación a la tensión y la negociación -en muchos casos mediatizada por la violencia- cristalizando como formas de diferenciación y jerarquización que regulan las relaciones entre pares. Así, llegamos a la etapa de educación secundaria donde los datos de la investigación revelan que 8 de cada 10 estudiantes ha presenciado burlas e insultos relativos a la orientación sexual y la identidad de género, casi la mitad del alumnado $(46,8 \%)$ ha sido testigo de situaciones de exclusión (como dejar de lado y evitar la interacción) y el 37,6\% afirma haber presenciado amenazas y expresiones de odio por los mismos motivos. Finalmente, si pensamos en una clase de 30 estudiantes, 6 habrían presenciado hechos de violencia física motivada por homofobia.

Como han señalado diversos autores (Leverenz, 1986; Blumenfeld, 1992; Parker, 1996; Kimmel, 1997; Borrillo, 2001; Pichardo, 2009), la homofobia no se dirige exclusivamente contra las personas LGBT, sino que también pueden sufrirla aquellas personas que con su expresión de género cuestionan las normas del sistema sexo/género. El término "fobia" no debe llevarnos a considerar la homofobia como una aversión o rechazo personal e irracional a la homosexualidad. La homofobia es una construcción social, histórica y cultural compleja cuya manifestación puede adquirir diversas formas: cognitiva, afectiva, conductual, liberal $e$ institucional (ver Borrillo, 2001; Pichardo, 2009), y que ha devenido en uno de los principales instrumentos de control de la 
masculinidad (Pichardo, 2012). Si anteriormente mencionábamos que la identidad masculina encuentra su antagonismo en la otredad femenina, la homofobia es el mecanismo por el cual se censura cualquier atisbo de feminidad en otros hombres y en uno mismo, ya que es la identidad masculina - $y$ no la femenina- la que se construye en el temor a ser o parecer homosexual (Kimmel, 1997) y, en consecuencia, en el rechazo tanto a la sexualidad no heterosexual como a las expresiones de género que cuestionan el sistema sexo/género.

Esto puede observarse en los resultados de la investigación, donde se pone de manifiesto que existe un porcentaje de alumnado heterosexual que sufre acoso por homofobia en sus centros educativos ${ }^{11}$. Una limitada conceptualización de la homofobia entendida como el mero rechazo a la homosexualidad puede invisibilizar estos casos e impedir a la comunidad educativa actuar a tiempo, lo que puede traer consigo graves consecuencias. Tal es así, que una chica de 15 años que al completar el cuestionario expresó tristeza y tensión a la hora de asistir a su colegio, completó el apartado de comentarios con el siguiente párrafo:

Por favor, acaben con el bullying. Es muy triste sentirme prisionera en mi propio cuerpo y pensar en el suicidio, intentarlo y luego tener que fingir. Me siento prisionera: me juzgan de lesbiana y me gustan los chicos, y tengo novio. Tener que hablar mucho para ocultarlo, llorar en silencio y vestir oscuro, esconder mis cortadas... Por favor, paren el bullying.

${ }^{11} \mathrm{El}$ acoso homófobo no se limita únicamente al alumnado heterosexual. Víctor Díez Mazo, profesor en el CEIP Valdés Leal (Sevilla), relata en una entrevista realizada durante el trabajo de campo: "Yo recuerdo en un centro educativo que un compañero tenía pluma, era heterosexual con pluma, pero tenía pluma. Y había alumnado, de quinto de primaria, que bueno, tuvieron unas reacciones que no eran las adecuadas". De hecho, de los 19 docentes que afirmaron en el cuestionario sufrir acoso homófobo por parte del alumnado, 11 son heterosexuales. Nota del autor: En España, se dice que tienen "pluma" las personas que performan una expresión de género considerada "femenina". 
El caso de esta chica forma parte del alumnado heterosexual que sufre acoso escolar debido a que su expresión de género no responde a la normatividad. Sin embargo, como se señala en el siguiente gráfico, el acoso escolar motivado por expresiones de género no normativas difiere también entre sexos: un $4,37 \%$ de chicos heterosexuales afirma haber sufrido burlas e insultos por parecer LGBT, mientras que la cifra se reduce prácticamente a la mitad en el caso de las chicas $(2,10 \%)$. También los hombres jóvenes bisexuales y homosexuales que sufren acoso por ser $\mathrm{o}$ parecer LGBT superan en porcentaje significativamente a las chicas bisexuales y lesbianas.

Porcentaje de alumnado por sexo y orientación sexual que ha sufrido acoso escolar por ser o parecer LGBT

$60,00 \%$

$50,00 \%$

$40,00 \%$

$30,00 \%$

$20,00 \%$

$10,00 \%$

$0,00 \%$

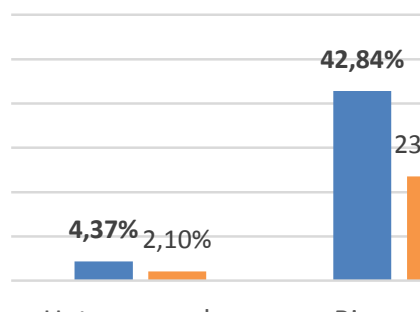

Heterosexuales

Bisexuales

Homosexuales
No lo sabe

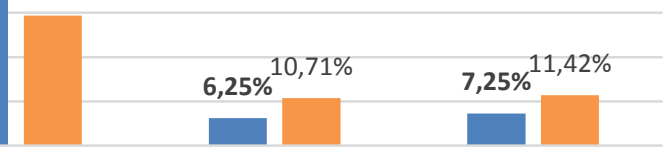

$51,27 \%$

$29,41 \%$

Prefiere no

contestar

Finalmente, cabe destacar que un $7,17 \%$ de hombres jóvenes heterosexuales señaló además haber sufrido burlas $e$ insultos por "hacer cosas de chicas", mientras que el porcentaje de chicas que sufrieron acoso por "hacer cosas de chicos" es menos de la mitad $(2,34 \%)$ que en el caso de los chicos.

El nivel de presión que ejerce la homofobia sobre las vidas y relaciones de los hombres es desmedido, y sin embargo, invisible. Al inicio del apartado anterior se ponía de relieve la aparente contradicción entre la percepción que tiene el alumnado sobre sus 
centros educativos como ambientes tranquilos y cooperativos y los datos que muestran la frecuencia del acoso escolar entre los hombres jóvenes. Esto responde a un proceso de invisibilización y normalización de la violencia estrechamente ligado al género. Existen violencias consideradas más o menos normales que encuentran mecanismos de legitimación diferentes: la violencia contra las mujeres en sus diferentes expresiones -física, psicológica, sexual, económica, simbólica-, las guerras, la violencia en el deporte, la expoliación del medio ambiente, la violencia de Estado, la desigualdad de clase, el racismo, etc. Todas ellas son perpetradas por una abrumadora mayoría de hombres y se ofrecen también de forma espectacularizada para el consumo del público masculino en una cultura mediática saturada de violencia, de tal forma que nos cuesta reconocerla como tal (Kaufman, 2009). Las desigualdades encuentran en la invisibilización y en la normalización a sus principales mecanismos de legitimación: no puede enfrentarse lo que no se percibe, no indigna lo que "siempre fue asî" (Pichardo, 2009).

Una investigación reciente sobre los efectos longitudinales de la exposición a la violencia entre niños y jóvenes señala que mientras mayor es la exposición a la violencia, mayor es también su consideración como algo normal y como una estrategia válida para resolver conflictos (Orue et al., 2011); y la masculinidad es, como hemos visto, sobre todo una experiencia de tensión $y$ conflicto. Teniendo en cuenta que los hombres jóvenes son quienes acosan en mayor medida, pero también quienes son acosados con mayor frecuencia, no sería extraño pensar que serán ellos quienes mayoritariamente invisibilicen y normalicen la violencia escolar. De hecho, en esta dirección apuntan los datos de la investigación: si bien la mayor parte del alumnado coincide en que el acoso escolar es un problema grave, existe un $36 \%$ del alumnado que justifica y relativiza el acoso. En el gráfico de las opiniones del alumnado sobre el acoso escolar, es al distinguir las respuestas por sexos que nos encontramos que las mujeres jóvenes superan en un $14 \%$ a los hombres jóvenes en las respuestas que consideran el acoso como algo grave y que no 
debería permitirse en ningún caso. Por otra parte, los hombres jóvenes duplican en porcentaje a las chicas en las respuestas que consideran el acoso escolar como algo que es "inevitable ya que hay personas que se lo merecen" (7\% frente a $3 \%$ ), como algo normal que "ocurre en todas partes" (10\% frente a 5\%), o como algo a lo que no hay que darle importancia porque "no hay mala intención" (9\% frente a 4\%). Como vemos, tal es el nivel de normalización del acoso escolar para los jóvenes, que llegan a considerar que no existe violencia en los insultos y burlas que intercambian con sus compañeros: "muchos insultos se han convertido en una forma amistosa de hablar y no hay que tomarlos como algo negativo" (Chico, 17 años); "la mayoría de las veces que nos vacilamos es sin ánimos de ofender" (Chico, 17 años).

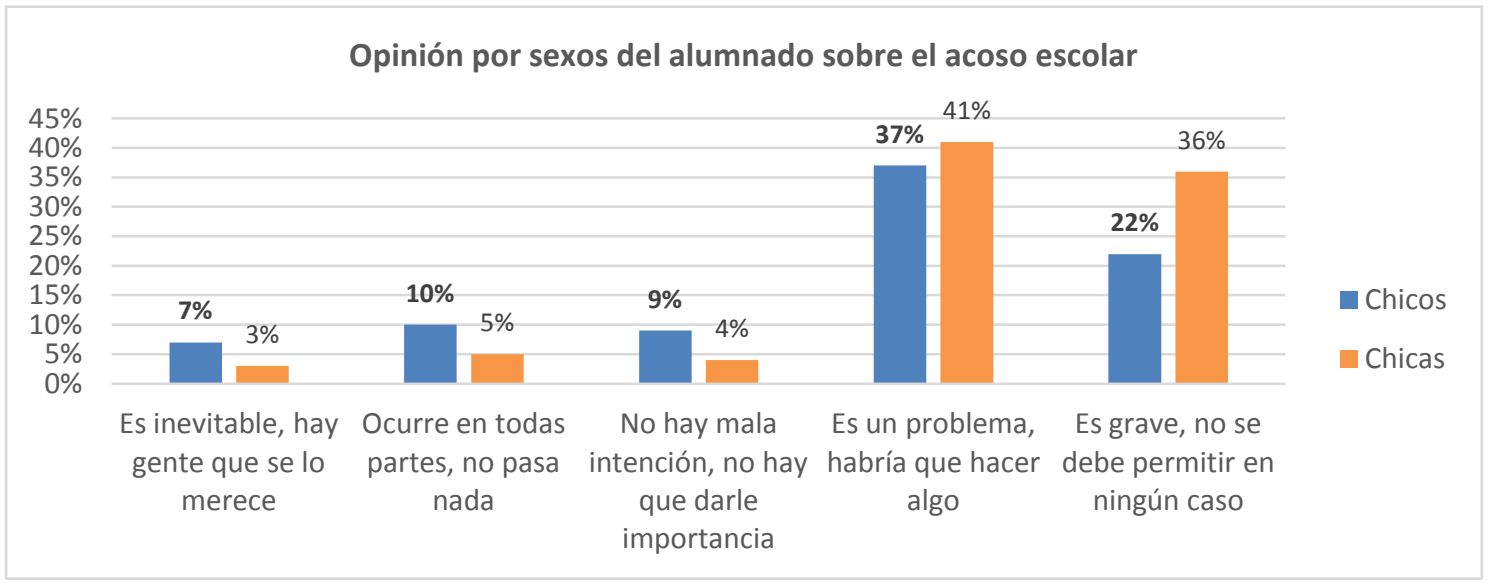


De acuerdo con Pichardo (2006:17-20), la invisibilización y la normalización son también características del acoso escolar por homofobia, ya que suele obviarse su carácter homófobo y se justifica por su continuidad y recurrencia a lo largo de los tiempos, casi como parte de una "tradición". Así lo constatan los discursos de estos chicos: "Yo saludo a amigos míos como 'iEy, marica!' y etc... Y respeto a los homosexuales" (Chico, 16 años); "Los insultos referentes a homosexualidad no se dicen en serio" (Chico, 15 años); "Se oye 'maricón', 'bollera' que, por ejemplo, hay veces que se dicen de forma inofensiva a un amigo tuyo también, no sólo como forma de insulto" (Chico, 15 años). Así, encontramos entre los jóvenes que "la homofobia se sigue considerando como un tipo de violencia normal o tolerable" (Pichardo, 2009:33).

Estos discursos, de aparente contradicción interna, son perfectamente coherentes si tenemos en cuenta el carácter vinculado a la masculinidad de la homofobia: en este caso, llamar "marica" a un amigo nos habla más de las configuraciones en las que la homofobia interviene en la construcción de la masculinidad en las relaciones entre varones heterosexuales que de la aversión o rechazo a los homosexuales. Es por eso que el segundo muchacho puede decir sinceramente que "los insultos referentes a la homosexualidad no se dicen en serio" y el último joven agregar que decir "maricón" a un amigo es "inofensivo", ya que la ofensa no radica concretamente en la orientación sexual, que puede ser "respetable" según el primer joven, sino simbólicamente en el desafío a la masculinidad.

Así, la invisibilización y normalización de la homofobia y de la violencia entre pares encuentra su intersección en las relaciones de dominación/subordinación de las masculinidades, ya que, como se señalaba en el apartado anterior, la hegemonía interna entre los hombres jóvenes se disputa constantemente, midiendo los límites de las masculinidades con violencia, pero, como veremos, también con miedo y silencio.

La homofobia atraviesa de forma transversal la vida de los y las jóvenes LGBT, de manera que no suelen contar o buscar ayuda en sus familias o amistades cuando sufren acoso escolar por 
homofobia, ya que si aún no se han visibilizado como LGBT, corren el riesgo de sufrir homofobia por parte de personas de su propio entorno (Pichardo, 2009). Esta especificidad difícilmente tenga lugar cuando las causas de acoso y discriminación tengan otros motivos, sin embargo, vemos en el siguiente gráfico que prácticamente la mitad $(49,60 \%)$ de los hombres jóvenes no cuenta a nadie que sufre acoso escolar, mientras que la cifra de mujeres se reduce a la mitad de los casos. Por otra parte, vemos que en caso de contarlo o pedir ayuda la proporción se invierte: las chicas prácticamente duplican a los chicos a la hora de contar las situaciones de acoso o buscar ayuda entre sus amistades y familiares.

Relación por sexo de jóvenes según a quiénes han contado o pedido ayuda cuando han sufrido acoso escolar

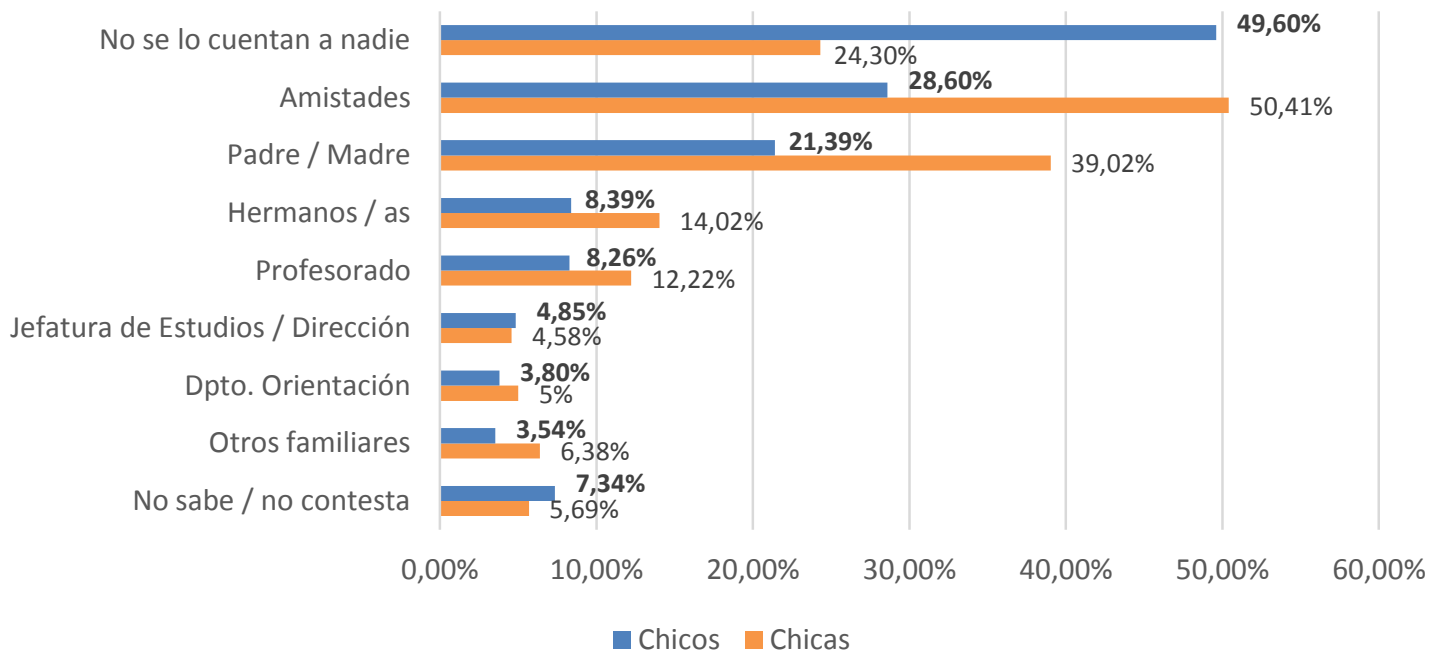

Si tenemos en cuenta la homofobia más allá de la sexualidad y como un mecanismo de control de la masculinidad, podemos entender que este silencio se debe a que los jóvenes, además de tener que estar constantemente demostrando su 
masculinidad, temen que cualquier signo de debilidad pueda ser usado en su contra (Seidler, 2006) y ser expuestos como vulnerables, cobardes, frágiles, poco "hombres". Como señala certeramente Kimmel (1997:10) "nuestros miedos son la fuente de nuestros silencios, y los silencios de los hombres son los que mantienen el sistema". Así, un joven homosexual víctima de acoso homófobo temerá recurrir a su familia o amistades por miedo a la homofobia, mientras que un joven heterosexual víctima de acoso temerá recurrir a su familia o amistades por miedo a ver cuestionada su masculinidad por el mismo motivo, la homofobia.

El miedo a sufrir homofobia articula el denominado "contagio del estigma" (Pichardo, 2006:19), que tiene lugar cuando las personas que se relacionan o apoyan a las víctimas de homofobia terminan por sufrir ellas mismas la violencia homófoba. Nuevamente, este tipo de situaciones no suelen darse con otras formas de acoso como el vinculado a las cuestiones físicas, raciales, étnicas o religiosas. Un joven blanco no temería sufrir racismo por defender a un joven negro de una agresión racista, de la misma manera, un joven nativo no temería sufrir xenofobia por defender a un joven inmigrante. Sin embargo, en una confrontación donde un joven -o un grupo de jóvenesheterosexual(es) cuestiona a otro(s) su masculinidad a través de burlas e insultos homófobos como "maricón" o "nenaza", el contagio de estigma puede impedir el apoyo o las alianzas de otros jóvenes heterosexuales que no quieran poner en riesgo su masculinidad. De este modo, la relación, amistad o mero apoyo a las personas LGBT, pero también a las víctimas de acoso homófobo que son heterosexuales, está castigada simbólicamente como una forma de control de la masculinidad vinculada a la heteronormatividad y la homofobia. De acuerdo con la teoría de Connell $(1995,2005)$ sobre las relaciones entre las masculinidades, este miedo al desprestigio funciona como un mecanismo de control que tiene como resultado la complicidad con el modelo de masculinidad hegemónica cuya posición dominante precisa de la subordinación de otras masculinidades subalternas, como las homosexuales. Este es un motivo más por el que -aún sin 
adecuarse a la masculinidad hegemónica- los hombres jóvenes heterosexuales irán aprendiendo a cuidar sus actitudes y palabras, a regular sus cuerpos y percepciones, para finalmente terminar enquistando sus dificultades para tender la mano, para ofrecer o buscar ayuda frente a la violencia. El temor al contagio del estigma tiene, además, como una de sus consecuencias directas, la perpetuación de la invisibilidad de la homofobia como un problema social que afecta a todas las personas e involucra a toda la comunidad educativa.

\section{Conclusiones}

Como hemos visto, la homofobia, como una forma específica de violencia, está estrechamente vinculada a la construcción, mantenimiento y control de la masculinidad $e$ interfiere no sólo en la vida de las personas LGBT, sino en el desarrollo de las relaciones de toda la comunidad educativa. El acoso escolar es una forma ritualizada de violencia con una gran eficacia social entre los hombres jóvenes, donde la homofobia juega un papel fundamental: a través del miedo, invisibiliza y normaliza la violencia, silencia y aísla a las víctimas y perpetúa la legitimidad de las burlas, insultos y amenazas como una forma válida de relación entre pares.

La introducción de una perspectiva crítica sobre la condición de género de los hombres es una cuenta pendiente tanto en el debate público como en el político, tanto en los diagnósticos de las causas de la violencia y la desigualdad como en los abordajes de sus consecuencias. Si los jóvenes recurren al acoso escolar como una forma de hacer género y recurren a la violencia para hacerse hombres, las estrategias para prevenir y erradicar el acoso escolar deben visibilizar las particulares intersecciones entre sexo, género y sexualidad que subyacen al acoso escolar.

Introducir en las aulas contenidos transversales con perspectiva de género, y específicamente con una perspectiva crítica sobre las masculinidades que incluya la diversidad sexual, familiar y de identidad de género, puede ayudar a reducir la 
violencia en los ámbitos educativos pero también a hacer visibles y accesibles formas alternativas de ser y hacerse hombre, para transformar paulatinamente las normas, valores y conductas que median las relaciones entre los hombres, sus relaciones con las mujeres, los niños y niñas, y con el conjunto de la sociedad y el medio ambiente. Así, los adolescentes tendrán al menos una oportunidad para transformarse y encontrar su lugar en los movimientos feministas y LGBT, a cuyas luchas $y$ victorias debemos la mera posibilidad de pensarnos como hombres.

\section{Referencias bibliográficas}

BLUMENFELD, Warren. Homophobia: how we all pay the price. Boston, Beacon Press, 1992.

BorRILlo, Daniel. Homofobia. Barcelona, Bellaterra, 2001.

ConNell, Raewyn. Masculinidades. México, PUEG, 1995.

CONNELL, Raewyn. Arms and the man: using the new research on masculinity to understand violence and promote peace in the contemporary world. In: BREINES, I.; CONNELL, R.; EIDE, I. Male roles, masculinities and violence. Paris, UNESCO, 2000, pp.21-33.

ConNell, Raewyn. Gender. Cambridge, Polity Press, 2002.

CONNELL, R.W.; Messerschmidt, J.W. Hegemonic masculinity: Rethinking the concept. Gender and Society, vol. 19, n 6, dec. 2005, pp.829859.

Del Barrio, C.; Martín, E.; AlmeidA, A.; Barrios, A. Del maltrato y otros conceptos relacionados con la agresión entre escolares, y su estudio psicológico. Infancia y Aprendizaje: Journal for the Study of Education and Development, vol. 26, n 1, 2003, pp. 9-24.

DE STÉFANO BARBERO, Matías. ¿Morir de éxito? Cuatro apuntes sobre los desafíos de los estudios sobre masculinidades ante el patriarcado contemporáneo. Revista Andariegas, n ${ }^{\circ}$ 3, 2015, pp.14-17. Enlace: http://issuu.com/comunicacion_social_ew/docs/andariegas3-1

De StÉfano Barbero, M.; PuChe, L.; Pichardo, J.I. El compromiso de la investigación social en la construcción de otra escuela posible. Revista Interuniversitaria de Formación del Profesorado. Vol. 29, $\mathrm{n}^{\circ}$ 
1, abril 2015, pp.46-90. Enlace: http://www.redalyc.org/articulo.oa?Id $=27439665004$

De StéfAno BARBero, M.; Pichardo , J.I. Sumando Libertades: Guía Iberoamericana para el abordaje del acoso escolar por homofobia y transfobia. Red Iberoamericana de Educación LGBTI, 2016. Enlace: www.educacionlgbti.org/wpcontent/uploads/2016/11/SumandoLibertades_Nov16.pdf

GARChITORENA, Marta. Informe Jóvenes LGTB. Madrid, FELGTB, 2009.

GENERELO, Jesús. (coord.) Acoso escolar homofóbico y riesgo de suicidio en adolescentes y jóvenes LGB. Madrid, COGAM/FELGTB, 2012.

Generelo, J.; Pichardo, J.I.; Galofré, G. (coords.) Adolescencia y sexualidades minoritarias. Voces desde la exclusión. Jaen, Alcalá Grupo Editorial, 2008.

GILMORE, David. Manhood in the making: Cultural concepts of masculinity. New Haven: Yale University Press, 1991.

GuASCH, Oscar. Héroes, científicos, heterosexuales y gays. Barcelona, Bellaterra, 2006.

HOOKS, Bell. The Will to Change: Men, Masculinity, and Love. New York, Atria Books, 2004.

Joel, D.; Berman, Z.; TAVOR, I.; Wexler, N.; Gaber, O.; Stein, Y.; Shefi, N.; Pool, J.; Urchs, S.; Margulies, D.; Liem, F.; Hänggi, J.; Jäncke, L.; Assaf, Y. Sex beyong genitalia: The human brain mosaic. In: PNAS vol. 112, n 50. DOI:10.1073/pnas.1509654112

KAUFMAN, Michael. The construction of masculinity and the triad of men's violence. In: ORE, T. (ed.) The Social construction of difference and inequality: race, class, gender, and sexuality. Boston, mcgrawHill, 2009, pp.584-598.

KEMPER, Theodore. Social Structure and Testosterone. New Brunswick, Rutgers University Press, 1990.

KIMMEL, Michael. Homofobia, temor, vergüenza y silencio en la identidad masculina. In: VALDES, T. Y OlAVARRÍA, J. (eds.) Masculinidad/es: poder y crisis. ISIS-FLACSO, Ediciones de las Mujeres $n^{\circ} 24,1997$, pp.49-62. 
LAGARDE, Marcela. Género y feminismo: desarrollo humano y democracia. Barcelona, Horas y horas, 1996.

Leverenz, David. Manhood, Humiliation and Public Life Some Stories. Southwest Review, vol. 71, n 4, 1986, pp.442-462.

Moreno, O.; PuCHE, L. (eds.) Transexualidad, adolescencias y educación. Miradas multidisciplinares. Madrid, Egales, 2013.

Orue, I.; Bushman, B. J.; Calvete, E.; Thomaes, S.; Orobio de Castro, B.; Hutteman, R. Monkey See, Monkey Do, Monkey Hurt: Longitudinal Effects of Exposure to Violence on Children's Aggressive Behavior. Social Psychological and Personality Science vol. 2, $\mathrm{n}^{\circ} 4$, 2011, pp.432-437.

PARKER, Andrew. The construction of masculinity within boys physical education. Gender and Education, vol. 8, n 2, 1996, pp.141-158.

PICHARDO, José Ignacio. Especificidad del acoso escolar por homofobia. In: Generelo, J., PiCHARDO, J.I. (coords.) Homofobia en el sistema educativo. Madrid, COGAM, 2006.

PICHARDO, José Ignacio (ed.) Adolescentes ante la diversidad sexual. Homofobia en los centros educativos. Madrid, Catarata, 2009.

PICHARDO, José Ignacio. El estigma hacia personas lesbianas, gays, bisexuales y transexuales. In: GAVIRIA, E.; GARCÍA-AEL, C.; MOLERO, F. (coords.) Investigación-acción. Aportaciones de la investigación a la reducción del estigma. Madrid, Sanz y Torres, 2012, pp.111-125.

PiCHARDO, J.I.; De StÉFANO, M. (eds.) Diversidad sexual y convivencia: Una oportunidad educativa. Madrid, Universidad Complutense de Madrid, 2015. Enlace: https://goo.gl/BH9uXZ

PICHARDO, J.I.; DE STÉFANO, M.; Faure, J.; Saenz, M.; Williams, J. Abrazar la diversidad: propuestas para una educación libre de homofobia y transfobia. Madrid: Instituto de la Mujer y para la Igualdad de Oportunidades, Ministerio de Sanidad, Servicios Sociales e Igualdad de España, 2015 [https://www.msssi.gob.es/ssi/igualdadOportunidades/noDiscriminacio n/documentos/Abrazar_la diversidad_v_d.pdf].

SEIDLER, Victor. Masculinidades. Culturas globales y vidas íntimas. Barcelona, Montesinos, 2006. 
SEIDLER, Victor. La violencia, ¿̇un juego de hombres? In: RAMÍREZ, J.; URIBE, G. (eds) Masculinidades. El juego de género de los hombres en el que participan las mujeres. Madrid, Plaza y Valdés, 2009, pp.113129.

SwaIN, Jon. Masculinities in Education. In: KIMMEL, M.; HEARN, J.; CONNELL, R.W. (eds.) Handbook of Studies on Men and Masculinity. Londres, SAGE, 2005, pp.213-229.

VIVEROS, Mara. La interseccionalidad: Perspectivas sociológicas y políticas. In: MAYORGA, C.; PeruchI, J.; PrAdo, M. (eds.) Olhares diversos: direitos sexuais, feminismos e lesbianidades. Belo Horizonte, Campagna, 2011. 